Biology | Ryoko Kuriyama

\section{The intricate world of the centrosome}

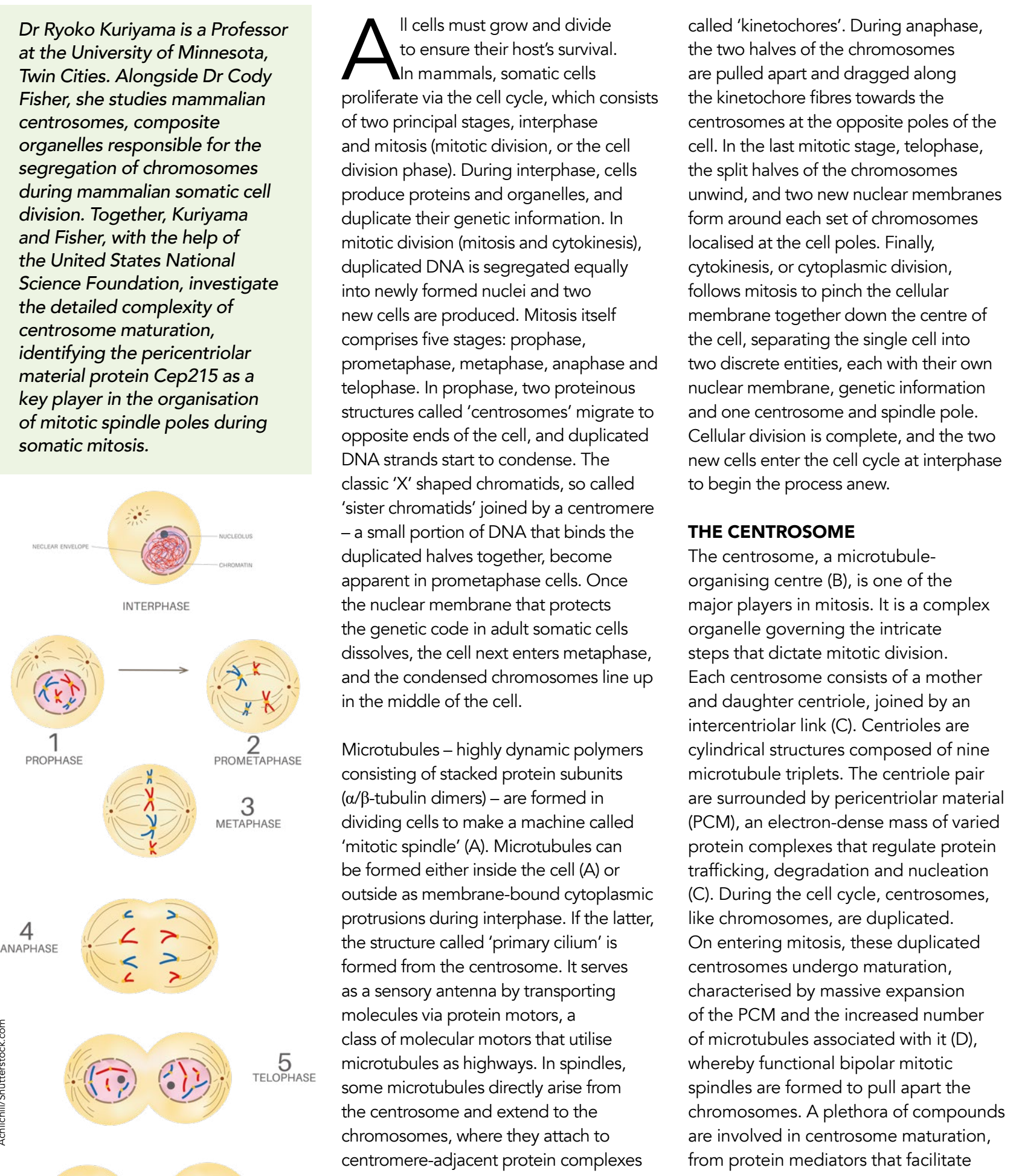

molecular interactions, to motor proteins hat dynamically build the spindle poles by transporting
structures.

\section{DIPLOID DISORDERS}

This tightly regulated molecular dance is essential for life, yet many of the mechanistic reactions involved in Understanding the basic science of thes interactions is essential, as aberrations in these processes can cause debilitating disorders, including cancers. Mistakes in centrosome maturation and subsequent chromosome segregation can also result in conditions such as microcephaly, a neurological disorder that hinders brain development, and primordial dwarfism, a genetic condition that impairs physical growth and intellectual development. (n) and DrCody Fisher at the University of Minnesota have been investigating centrosome maturation, aiming to shed light on the roles and importance of proteins within the PCM. They believe that understanding the basic molecular mechanisms behind centrosome maturation will pave the way to medical intervention for aneuploidy-derived diseases, ultimately improving, and saving, lives.

\section{CEP215: THE KEY PLAYER?}

Of particular interest to Professor Kuriyama and collaborators is the PCM protein Cep215. Mutations in Cep215 but how this prouin functions centrosome maturation, and why it is so essential to normal brain development. is still unclear. Homologs of the mammalian Cep215 protein appear widely across species, and such highlevel genetic conservation indicates that this protein must play an important role in maintaining species' health and longevity. Cells lacking fully functioning Cep215 often display disconnected spindle poles and lack general microtubule organisation. Using a series of experiments, Kuriyama and her team set out to pinpoint the structure, within the mitotic cell cycle.

\section{STRUCTURE SOLVING}

To explore the structure of Cep215, the Kuriyama Lab examined the binding

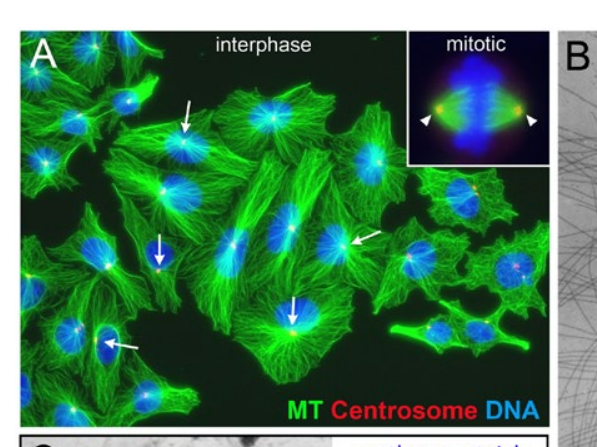

$B+1$

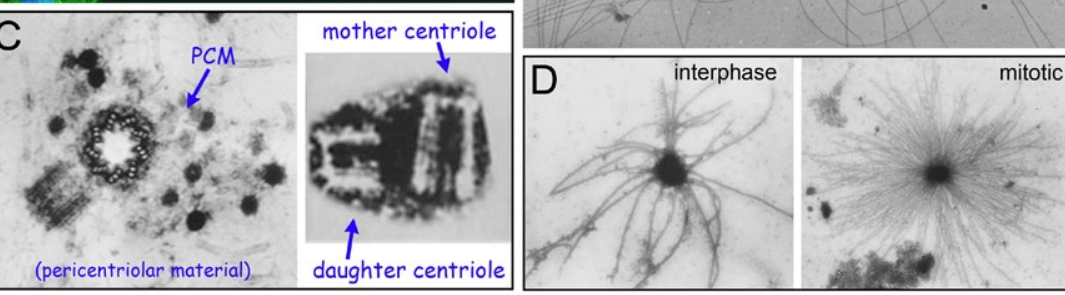

A. Microtubules (MTs; green) in interphase and mitotic cells (inset) seen by fluorescence microscopy.
Centrosomes (red) are located at the focal point of the interphase MT network (arrows land mitotic Centrosomes (red) are located at the focal point of the interphase MT network (arrows) and mito
spindle poles (arrowheads). DNA-containing nuclei and mitotic chromosomes are labelled blue. B. The centrosome is a microtubule-organising centre. Electron microscopy shows in vitro
polymerisation of microtubule onto the centrosome by incubation of isolated centrosomes with microtubule subunit proteins, tubulins.
c. Centrosomes are made of a ari or barrel-shaped centrioles and a surrounding fuzzzy
mass called 'pericentriolar material'. Mother and daughter centrioles are generally located

D. Centrosome to
centrosomes.

domains along the length of the protein. They cut Cep215 into a series of short $215 \mathrm{~N}$ (E). By fluorescence labelling this peptide and visualising it within hamster cells, the team were able to nactive (non-phosphory, pAu, AurA) against both forms of the kinase they found that localisation of pAurA overlapped significantly with that of $215 \mathrm{~N}$ at the centrioles, particularly

This tightly regulated molecular dance is essential for life, yet many of the

mechanistic reactions involved in centrosome maturation remain elusive.

centrosome during mitosis. The strong fluorescence intensity of the $215 \mathrm{~N}$ signal suggested to the researchers that $215 \mathrm{~N}$ plays an essential role in targeting the centrosome.

To help elucidate the function of the $215 \mathrm{~N}$ domain at the centrosome, the team chose to explore its interactions with other molecules known to be involved in centrosome maturation. They previously reported close relationship between fly homolog of Cep215 and Aurora A (AurA), thus this protein kinase, required for spindle organisation during exists within the mitotic cell in both around the proximal end of the mother centriole. The team hypothesised that, due to such significant overlap, these two molecules may interact at depletion experiments. They used which short double stands of RNA are used to inhibit expression of the target gene, to first deplete mitotic pAurA, f $215 \mathrm{~N}$, which rest then depleted 215N, levels of $\mathrm{A}$ Aur. that 215N a a. These results indicated that $215 \mathrm{~N}$ and pAurA are mutually localisation to the centrosome during maturation. this location, and investigated this by RNA interference (RNAi), a method in 


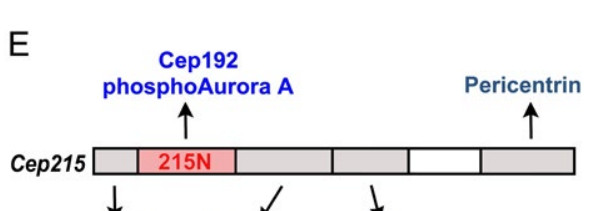

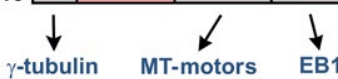

E. Cep215 consists of different domains binding lo specific molecules. An ewly identified
domain $215 \mathrm{~N}$ ) interacts with Cep 192 and

phosphoAurora A.
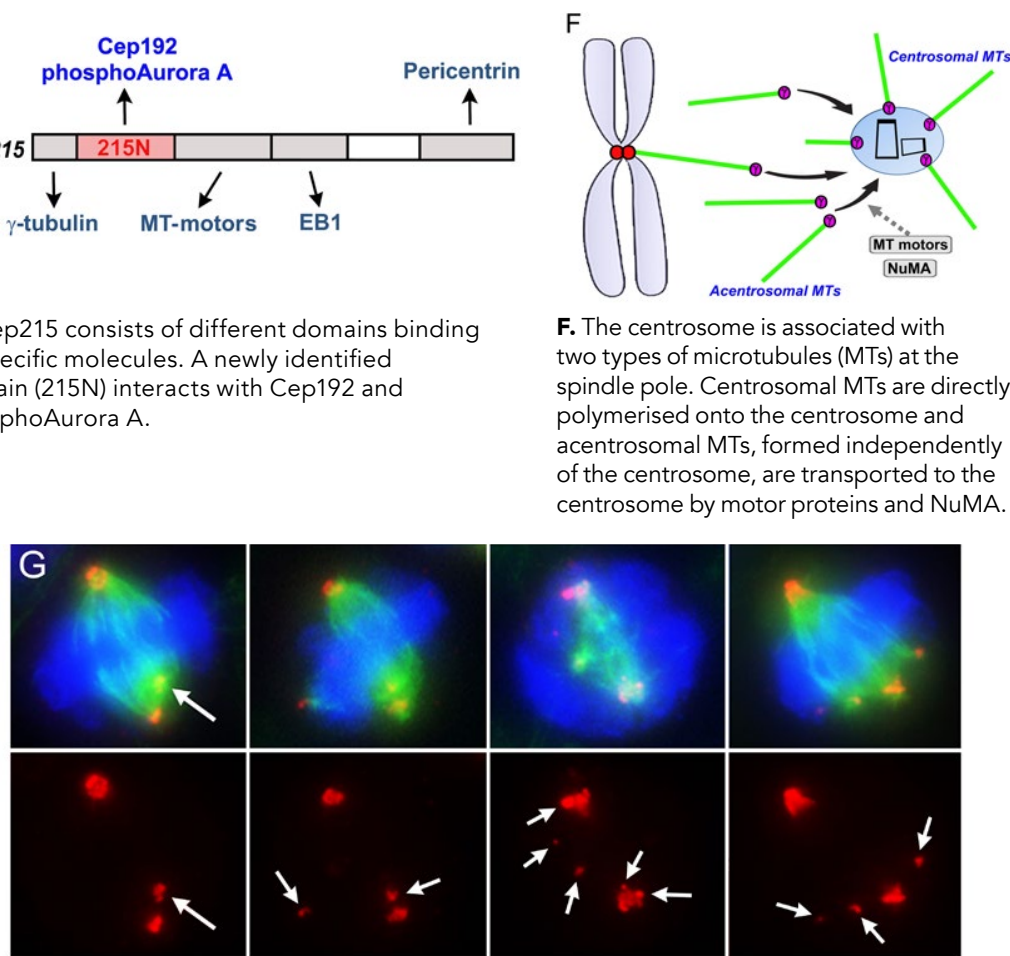

F. The centrosome is associated with
two types of microtubules (MTs) at the spotydle pole. Centrosomal MTs are directly
solymerised onto the centrosome and polymerised onto the centrosome and
acentrosomal MTs, formed independently of the centrosome, are transported to thy

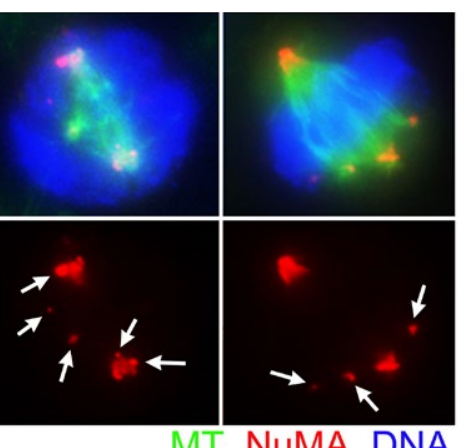

\section{PROTEIN CODEPENDENCY} After the interdependency of $215 \mathrm{~N}$ and pAurA was confirmed, the team decided to explore whether $215 \mathrm{~N}$ interacts with any other PCM proteins. They picked Cep192, a centrosome protein known to be involved in centriol duplication and microtubule nucleation onto the centrosome. By repeating their fluorescence localisation experiments, the team found that Cep192 and 215N co-localised identically at the centrosom In repeating their RNAi depletion cells with depled $192,215 N$ was

a similar role in forming spindle poles, by looking at the relationship between nucleating protein of the centrosomes. The researchers used RNAi to deplete 215N Cep215 in mitotic cells and looked at the abundance of $y$ tubulin, finding that up to $65 \%$ of $\gamma$-tubulin was retained in $215 \mathrm{~N}$ depleted cells in comparison to control with $215 \mathrm{~N} / \mathrm{Cep} 215$. This is in striking contrast to Cep192-depleted cells wher almost all $\gamma$-tubulin ( 93\%) disappears at the mitotic centrosome, indicating Cep215 clearly has only ar

Studies like Prof Kuriyama's demonstrate the value and gravity of investigating basic biological mechanisms.

undetectable at the centrosome. However,
when $215 \mathrm{~N}$ was depleted, Cep 192 still when $215 \mathrm{~N}$ was depleted, Cep192 still localised well to the centrosome, ind whilst $215 \mathrm{~N}$ and pAurA are mutually dependent, only $215 \mathrm{~N}$ is dependent upon
Cep192. These three components are Cep192. These three components are
physically interacting with each other (E).

As the team had confirmed a relationship between the spindle-pole building protein investigated whether Cep215 played
After showing that $215 \mathrm{~N}$ interacted with proteins essential for microtubul formation during centrosome in polymerisation of centrosomat microtubules, the team sought to elucidate its true role in centrosome maturation. They did this by examining the phenotypes of mitotic cells depleted of the whole protein, Cep215. In cells range of spindle pole abnomities, from cells with short, thin, or singular spindles, to those with knots of

To examine these spindle knots, the team looked at the distribution of nuclear mitotic apparatus protein (NuMA) which plays a role in transporting microtubules assembled independently of the centrosome (acentrosomal microtubules) coalescing one of their ends into the pole and attaching spindle-pole fibres to the centrosome (F). Due to multiple unfocused spindle ends, where NuMA is present, disorganised spindle knots are likely to have highly randomised distribution of NuMA (arrows in G). This hypothesis was confirmed - the researchers noted that in cells depleted of Cep215 alone, as well as triple-depleted of Cep215, Cep192 and pAurA, an increased number of randomly Kuntriced

results indicated that Cep 215, Cep192 pAurA act together as a protein complex in bringing the end of two types of MTs, centrosomal and acentrosomal, together by ensuring focused distribution of NuMA at the centrosome of each spindle pole ( $P$, and therefore correct attachment of each pole to the centrosome.

Based upon these results, the authors proposed that the major function of Cep215 is to organise the functional spindle poles by connecting the mitotic centrosome with the spindle poles, via interaction of its domains, $215 \mathrm{~N}$ being on

\section{BASIC CELL RESEARCH:}

This study serves to prove the complexity cells. Centrosome maturation itself is just a small, but significant, process within on phase of mitosis, which itself is a stage of the wider cell cycle. Small changes in any molecules within this stage, and the cycle as a whole, can have huge impacts on human development, as exemplified by the disorders microcephaly and primordial dwartism. If we don't understand these cellular processes deeply, we have no way of taking preventative actions against some of life's most debilitating disorders. Studies like value and Kravity of dinmonstrate the vilue and gravity of investiga

\section{Behind the Research}

Prof Ryoko Kuriyama

E: kuriy001@umn.edu T: +1 651-645-3049 T: +1 612-624-4966 W: https://cbs.umn.edu/contacts/ryoko-kuriyama-ph

\section{Research Objectives}

Research in Professor Kuriyama's laboratory centres on the cell cycle and cell growth control in mammalian current efforts are orientated towards understanding the molecular mechanism and regulation of mitosis and cytoplasmic division.

\section{Detail}

\section{Address}

Ryoko Kuriyama

Department of $G$

f Minnesota, Minneapolis,

Ryoko Kuriyama is Professor emeritus at the University of Minnesota, Twin Cities. After completion of her Ph.D. in Biochemistry (University of Tokyo, Japan), she moved to the University of Wisconsin-Madison (Molecular Biology Laboratory) to study mammalian centrosomes School as a faculty member.

Funding

ational Science Foundation, USA

Collaborators

Cody Fisher (University of Minnesota)

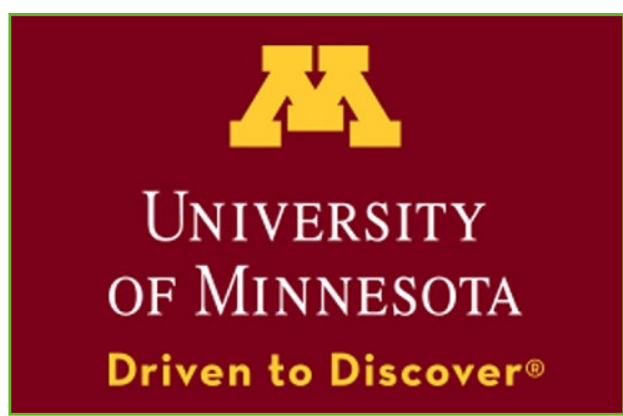

\section{References}

- Kuriyama, R. \& Fisher, C. (2020) A novel mitosisspecific Cep215 domain interacts with Cep192 and phosphorylated Aurora A for organization of spindle poles. Journal of Cell Science. 133, jcs240267, 1-21. - Jaiswal, S. \& Singh, P. (2021) Centrosome dysfunction in human disease. Seminars in Cell and Developmenta - Biology. 110, 113-122.

- Chavali, Petal. (2016) A CEP215-HSET complex links clustering in cancer. Nature Communications. 7, 11005, 1-16.

- Nigg, A. E. \& Raff, J. W. (2009) Centrioles, Centrosomes, and Cilia in Health and Disease. Cell. 139, 662-678. - Bornens, M. \& Gonczy, P. (2014) Centrosomes back in the limelight. Philosophical Transactions of the Royal Society B. 369, 20130452, 1-16.

- Terada, Y., Uetake, Y. \& Kuriyama, R. (2003) Interaction of Aurora-A and centrosomin at the microtubule763.

- Kuriyama, R. \& Borisy, G.G. (1981) Mirotubule-nucleating activity of centrosomes in Chinese hamster ovary cells isindependent of the centriole cycle but coupled to the mitotic cycle. J. Cell Biol. 91, 822-826.

\section{Personal Response}

Your research discovered a novel binding domain $215 N$, on the Cep215 protein. Do you think this
protein harbours further undiscovered domains th may help further elucidate its function?

II Yes, absolutely. Protein-protein interaction is a key because the integrity of the genetic code is at stake. To execute this process seamlessly, spindle components tempt int ty temporally- and spatially-dependent manner. Besides
$215 \mathrm{~N}$, Cep215 contains domains capable of associating with molecular motors and pericentrin, a scaffolding protein in the PCM. These associations suggest that Cep215 recruits acentrosomal microtubules to centrosomes and that it helps to organise the expanding
PCM structure. By identifying additional domains and their specific binding partners, we will have a better understanding of how Cep215 works in mitotic cells. 